\section{IN THE NEWS}

Genetics hits the headlines Much has been made of the release of the completed human genome sequence, which was heralded as a "cosmic triumph for biology" (Washington Post), and of the 50th anniversary of the DNA double helix, which "marked a turning point in history and sparked a revolution in molecular biology that continues to change the world today" (The Guardian).

Indeed, the impact of genetics on society is obvious in the diversity of genetics-related articles. "A body is to be exhumed for DNA testing" (The Times) in a highprofile paternity case to test the claims of five Italians on the $£ 300$ million estate left by Harold Acton to New York University. In the United States, the death of a nine month-old baby with a treatable, but undiagnosed, genetic disease has prompted a federal advisory panel to debate whether to "increase the list of diseases considered a minimum for newborn testing in every state" (Associated Press). Negative genetic modification (GM) publicity continues to crop up on both sides of the Atlantic, as a biotech company was fined when GM corn "contaminated nearby crops on Hawaii" (New York Times) and a 12-strong panel of the British public viewed GM foods "as unnecessary and not worth the risks to public health" (The Times). The successful sequencing of the severe acute respiratory syndrome (SARS) virus, described by Julie Gerberding as "a critically important step" (New York Times), also made headline news. The million dollar question is "in this amazing age of molecular genetics and medicine ... can scientists stop it?" (New York Times).

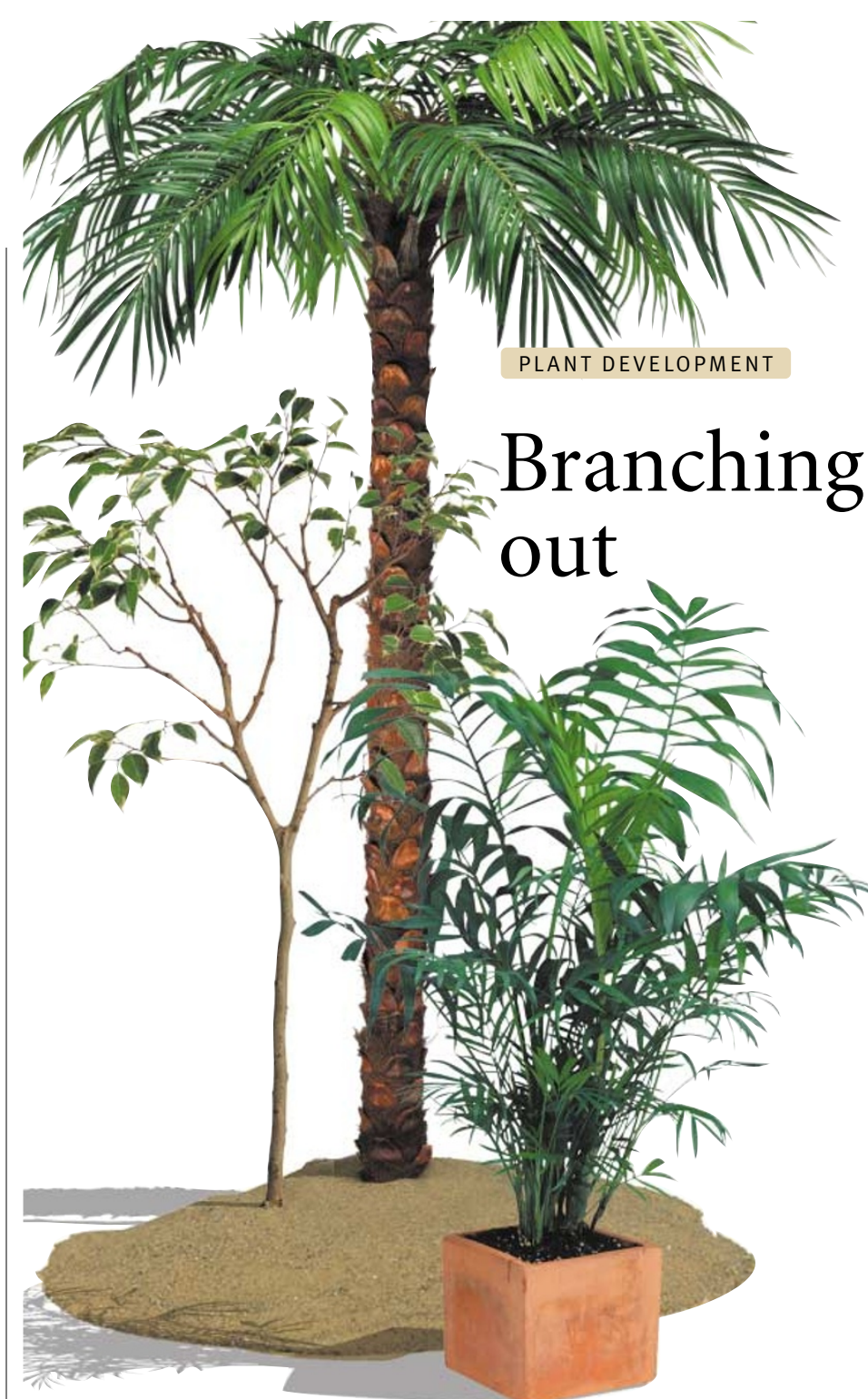

POPULATION GENETICS

\title{
They grow up so fast
}

From Adam and Eve to Genghis Khan, genetic inferences about human history provide some of the field's biggest headlines, but just how realistic are the assumptions on which such studies are based? Agnar Helgason and colleagues show that the answer is 'not very' in the case of the comparative rates of genetic drift for exclusively maternally or paternally inherited genes.

Large-scale studies that look at how a contemporary population is linked to successive generations of ancestors are rare, despite the fact that they are the best way to test the standard demographic models that are used in population genetic analyses. Helgason and colleagues have now done the 'granddaddy' of all such studies by tracing back the matrilineal and patrilineal ancestry of the 131,061 Icelanders that were born after 1972 .

Using a genealogical database that includes all living Icelanders, matrilines and patrilines were traced back from contemporary females and males, respectively, to ancestral cohorts. The authors used coalescent analyses of these genealogies to show that contemporary genes that are exclusively maternally (mtDNA) or paternally (Y chromosomes)
From daffodils to oak trees, the diversity in plant architecture is enormous, and different branching patterns underlie much of this variation. Now, Greb et al. in Arabidopsis, and Li et al. in rice, have identified a regulatory gene that is needed for branch formation throughout the flowering plants.

Branches are secondary shoots that grow from axillary meristems mitotic cells that are located in leaf axils. There has been considerable interest in the mechanisms that control the formation of axillary meristems and determine whether or not they grow out into branches. The tomato lateral suppressor (ls) mutant, fails to produce branches during leafproducing vegetative development, and so provides a good starting point to study axillary meristems. With this in mind, Greb et al. identified the Arabidopsis orthologue of $L S$, which they call LATERAL SUPPRESSOR (LAS). Like the tomato ls mutant, transposon-generated las mutants do not produce branches during vegetative growth, but do so in later reproductive development. Indeed, the similarity between the genes is high enough that the wild-type Arabidopsis LAS gene can rescue the tomato ls mutant.

inherited have originated from relatively few ancestors. For example, $61.8 \%$ of the mtDNA in the contemporary female population comes from just $6.6 \%$ of the females that were born between 1698 and 1742 .

Moreover, it emerged that contemporary Icelandic women have fewer matrilineal ancestors than the men have patrilineal ancestors, and that there is greater variation in the number of matrilineal descendants from female ancestors than in patrilineal descendants from male ancestors. This implies a greater rate of genetic drift for mtDNA than for Y chromosomes. The main cause of this difference is the shorter average time between female generations compared with male generations, and a stronger correlation in reproductive behaviour in the female line. 
H I G H L I G H TS

Although rice might look different from tomato and Arabidopsis, there are similarities in the way that they grow. The main stem produces leaves with associated axillary meristems, and the meristems that are closest to the base can develop into tillers - grain-bearing branches. Li et al. found a spontaneous mutation that causes a loss of vegetative axillary meristems and, therefore, tillers, as well as a reduction in plant height and reproductive branching. The mutated gene - MONOCULM1 (MOC1) - is homologous to LS. So, it seems that $L S$ gene function is widely conserved.

RNA in situ hybridization analysis showed that $L A S$ is expressed in the cells from which axillary meristems develop, whereas MOC1 is transcribed in axillary meristems from the earliest stages of development. Mutant analysis indicated that, in leaf axils, $M O C 1$ is genetically upstream of the known rice meristem regulators $\mathrm{OSH} 1$ and OsTB1. Similarly, LAS is needed in Arabidopsis for the correct expression of SHOOTMERISTEMLESS (STM) and REVOLUTA (REV) important genes that are required for meristem function. LAS and

These results are stunning, as a standard assumption has been that $\mathrm{Y}$ chromosomes drift faster than mtDNA because males are more promiscuous and vary more in their reproductive success than do females. If the results are replicated in other populations the implications could be important - more realistic estimates of the rates of genetic drift might completely change our interpretation of human mtDNA and Y-chromosome data.

One thing is for sure: in Iceland, at least, it seems that girls do grow up faster than boys!

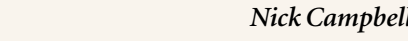

(2) References and links ORIGINAL RESEARCH PAPER

Helgason, A. et al. A population wide

coalescent analysis of Icelandic matrilineal and patrilineal genealogies: evidence for a faster evolutionary rate of mtDNA lineages than $Y$ chromosomes. Am. J. Hum. Genet. 72, 29 April 2003

WEB SITE

deCODE Genetics: http://www.decode.com
MOC1 might activate transcription directly as they are members of the plant-specific GRAS family, which are believed to be transcription factors. Consistent with this, Li et al. show that a MOC1-GFP fusion protein is localized in the nucleus.

The high level of conservation of $L A S$ and $M O C 1$, together with their upstream positions in known genetic hierarchies, indicates that they might be master regulators of axillary meristem formation. Therefore, these genes are likely to be a hot area for future research. Not only will this work contribute to our understanding of plant development, but it might also have an important agronomic impact because tiller production in cereals is a key determinant of grain yield and the level of tomato branching can affect fruit size.

Catherine Baxter

\section{(2) References and links} ORIGINAL RESEARCH PAPERS $L i, X$. et al. Control of tillering in rice. Nature 422, 618-621 (2003) | Greb, T. et al. Molecular analysis of the LATERAL SUPRESSOR gene in Arabidopsis reveals a conserved control mechanism for axillary meristem formation. Genes Dev. 17 1175-1187 (2003)

\section{$1175-1187(200$
WEB SITES}

Jiayang Li's laboratory:

http://www.genetics.ac.cn/xywwz/Faculty/jyli.htm Klaus Theres' laboratory:

http://www.mpiz-koeln.mpg.de/ theres

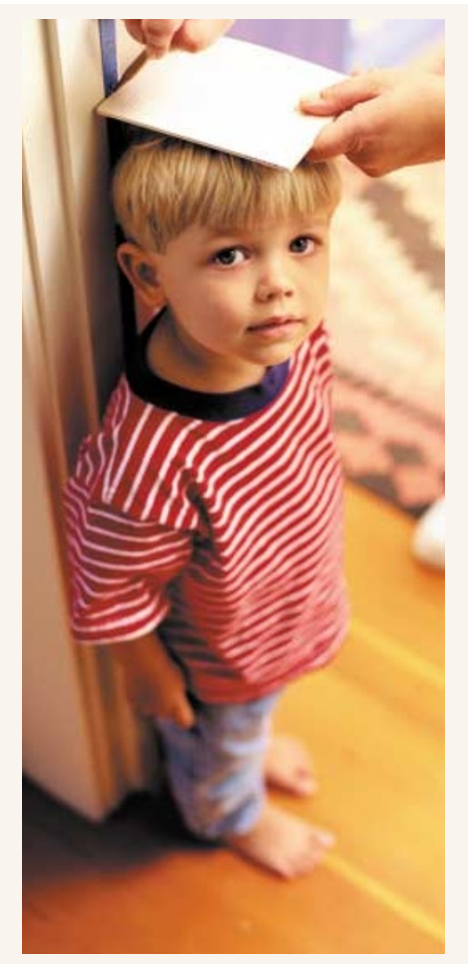

\section{IN BRIEF}

\section{FUNCTIONAL GENOMICS}

Genomic gene clustering analysis of pathways in eukaryotes.

Lee, M. J. \& Sonnhammer, E. L. L. Genome Res. 13, 875-882 (2003)

Although operons are rare in eukaryotes, genes that act in the same pathways might still be clustered. The authors looked for clustering in five eukaryotic genomes for genes that have been assigned to the same pathway in the KEGG database. Although genes associated with $30-98 \%$ of pathways cluster to some extent, surprisingly, there is little conservation between genomes as to which genes are clustered, perhaps reflecting lineage-specific evolutionary-events.

\section{FUNCTIONAL GENOMICS}

\section{Discovering novel cis-regulatory motifs using} functional networks.

Etteiller, L. M. et al. Genome Res. 13, 883-895 (2003)

Data from DNA-binding experiments, microarrays and genome comparisons have been used to facilitate regulatory-motif discovery. These authors use protein-protein interactions and metabolic networks in combination with Saccharomyces cerevisiae genome data to predict upstream regulatory motifs. They describe 42 potential sites, some of which are new and are associated with genes that are probably co-regulated. Their results show that interacting proteins are often transcriptionally coordinated, even if there is no evidence for their co-regulation in gene-expression data sets.

\section{MOUSE MODELS}

Mutations in dynein link motor neuron degeneration to defects in retrograde transport.

Hafezparast, M. et al. Science 300, 808-812 (2003)

The genetic basis of most motor neuron diseases (MND) is unknown. But a promising breakthrough has been made from the study of two mouse mutants - Legs at odd angles and Cramping 1. These mice have progressive neurodegenerative disorders and share a similar pathology to human MND. Both mutants carry a missense point mutation in the cytoplasmic dynein heavy chain, which is needed for microtubule-mediated retrograde transport in axons.

\section{CLONING}

Molecular correlates of primate nuclear transfer failures. Simerly, C. et al. Science 300, 225-227 (2003)

So far, no one has successfully cloned a primate, let alone a human, by nuclear transfer. Cloned rhesus monkey embryos fail to develop beyond early stages because many of the cells are aneuploid as a result of aberrant mitotic-spindle formation. Two proteins, NuMA and HSET, are needed for correct spindle organization, but in primates unlike other mammals - these proteins are exclusively associated with the chromosomes in unfertilized egg cells. As these chromosomes are removed during nuclear transfer, spindle organization is disorganized in primates and chromosome segregation fails. 